\title{
Stiff Fluid in Accelerated Universes with Torsion
}

\author{
Almaz Galiakhmetov \\ Department of Physics, Donetsk National Technical University, Kirova Street 51, Gorlovka 84646, Ukraine \\ Correspondence should be addressed to Almaz Galiakhmetov; agal17@mail.ru
}

Received 16 February 2013; Accepted 18 March 2013

Academic Editor: Sergei Odintsov

Copyright (C) 2013 Almaz Galiakhmetov. This is an open access article distributed under the Creative Commons Attribution License, which permits unrestricted use, distribution, and reproduction in any medium, provided the original work is properly cited.

The flat Friedmann universes filled by stiff fluid and a nonminimally coupled material scalar field with polynomial potentials of the fourth degree are considered in the framework of the Einstein-Cartan theory. Exact general solution is obtained for arbitrary positive values of the coupling constant $\xi$. A comparative analysis of the cosmological models with and without stiff fluid is carried out. Some effects of stiff fluid are elucidated. It is shown that singular models with a de Sitter asymptotic and with the power-law $\left(t^{4 / 3}\right)$ asymptotic at late times are possible. It is found that $\xi=3 / 8$ is a specific value of the coupling constant. It is demonstrated that the bouncing models without the particle horizon and with an accelerated expansion by a de Sitter law of an evolution at late times are admissible.

\section{Introduction}

Recent cosmic observations [1-6] favor an isotropic spatially flat Universe, which is at present expanding with acceleration. The source of this expansion is an unknown substance with negative pressure called dark energy (DE). Establishment of the origin of DE has become an important problem. Different theoretical models of DE have been put forward (see, e.g., the reviews [7-10] and references therein). Among these models various modifications of general relativity (GR) were considered, the Einstein-Cartan theory (ECT) in particular [11-13]. This theory [14-17] is an extension of GR to a space time with torsion, and it reduces to GR when the torsion vanishes. The ECT is the simplest version of the Poincaré gauge theory of gravity (PGTG). It should be noted that the ECT contains a nondynamic torsion, because its gravitational action is proportional to the curvature scalar of the Riemann-Cartan space-time. In this sense, the ECT is a degenerate gauge theory [17-20]. This drawback is absent in the PGTG since its gravitational Lagrangian includes invariants quadratic in the curvature and torsion tensors. Nevertheless the ECT is a viable theory of gravity whose observational predictions are in agreement with the classical tests of GR, and it differs significantly from GR only at very high densities of matter [17, 21, 22].

The ECT finds applications in cosmology [23-28], particle theory $[19,29,30]$, and the theory of strong interactions [31, 32]. From some time past, the interest to ECT has grown in connection with the fact that torsion arises naturally in the supergravity [33-35], Kaluza-Klein [36-38], and syperstring [39-41] theories. $f(R)$ gravity with torsion has been developed [42-45] as one of the simplest extensions of the ECT. In [43] it has been demonstrated that, in $f(R)$ gravity, torsion can be a geometric source for the accelerated expansion. The equivalence between a Brans-Dicke theory with the Brans-Dicke parameter $\omega_{0}=-3 / 2$ and the Palatini $f(R)$ gravity with torsion has been demonstrated in [44]. $f(T)$ gravity has been constructed [46-48] as the extension of the "teleparallel" equivalent of GR [49], which uses the Weitzenböck connection that has no curvature but only torsion.

At present, the considerably increased precision of measurements in the modern observational cosmology stipulated its essential progress. In this connection, the exact cosmological solutions, which makes it possible to elucidate the detailed picture of an evolution of models, are of great interest. It is well known that in GR the exact solutions of the Friedmann-Robertson-Walker (FRW) cosmological models constitute a basis for comparison of theoretical predictions with observations. On the other hand, the problem of the cosmological perturbations may be correct, if it is constructed against a background of the exact solution.

Other problems of the cosmology, singularities, horizons, and so forth, remain no less acute problems. The main aim of this paper is the investigation of the possibility of the solution 
of some problems of the modern cosmology in the framework of the ECT. Furthermore, the exact integration of the ECT equations for the different combination of sources and the comparative analysis of the corresponding cosmological models are of interest in their own right, since they allows to elucidate the role of the sources of the gravitational field in cosmology.

In the framework of the ECT with a nonminimally coupled material scalar field that has polynomial potentials of the fourth degree, we here study isotropic spatially flat cosmologies in which stiff fluid is taken into account. The motivation to investigate a nonminimally coupled scalar field is based on the results obtained within the framework of the torsionless theories of gravity in connection with the inflationary cosmology (see, e.g., [50-54]) and DE model building [55-57]. On the other hand, the consideration of this version of the ECT in comparison to $f(R)$ gravity with torsion and $f(T)$ gravity is motivated by the fact that, in my opinion, the ECT requires a further study. It is relevant to remark here that, asit is well known, in the literature there exist a limited number of the exact cosmological solutions in the framework of the ECT.

The interest in a scalar field potential $V(\Phi)$ in relativistic theories of gravity has been aroused by a number of circumstances: its role in cosmology with a time variable cosmological constant [58] and in quantum cosmology [59]; models with $V(\Phi)$ arise in alternative theories of gravity $[60,61]$ and supergravity [62]; a scalar potential governs an inflation [63] and is actively used in theories of dark matter $(\mathrm{DM})$ and dark energy (DE) [7-10]. It is well known from GR that a period of accelerated expansion requires $V(\Phi)>$ 0 . In the paper we will consider cosmological models with $V(\Phi)>0$ and $V(\Phi)<0$. The reasons to study cosmology with negative potentials were considered in [64].

A stiff cosmological fluid, with pressure equal to the energy density, can be described by a massless scalar field, which is predicted by the string theory. On the other hand, the stiff fluid is an important component because, at early times, it could describe the shear dominated phase of a possible initial anisotropic scenario and is dominating the remaining components of the model [65]. Of no little interest is the circumstance that this fluid leads to integrability of the Einstein-Cartan equations.

In [66], the flat Friedmann universes filled by radiation, stiff fluid and a nonminimally coupled ghost scalar field with polynomial potentials of the fourth degree $V(\Phi)$ have been investigated in the framework of the ECT. Exact particular solutions have been obtained and analyzed. The role of sources in the evolution of models has been elucidated.

The exact general solution of an analogous problem for models containing only a nonminimally coupled scalar field with an arbitrary coupling constant $\xi$ has been obtained in [12]. An analysis of the solutions for the material scalar field has shown the following.

(i) For $\xi>0$ the solution exists for $\xi \geq 1 / 6$, $\Phi^{2}<(\kappa \xi)^{-1 / 2}$, where $\kappa$ is Einstein's constant only and describes a countable number of nonsingular cosmological models with de Sitter asymptotics for the late times.

(ii) For $\xi<0$ the five types of singular models exist: for the first model the scale factor $a(t)$ as $t \rightarrow \infty$ grows by the law $a \sim t^{p}(p<1)$; for the second model $a(t)$ asymptotically tends to a finite value; other three models expand from an initial singularity, reach the maximum, and then begin to contract to a final singularity.

(iii) There are the specific values of the parameter $\xi$ : $1 / 6,-1 / 6$, and $-3 / 2$. The specific feature of the value $\xi=1 / 6$ consists in the behavior of the scale factor, when $\left(a-a_{\min }\right) / a_{\min } \ll 1: a \simeq a_{\min }(1+y)$, where $y \sim t^{3}$ for $\xi=1 / 6$, while $y \sim t^{2}$ for $\xi>1 / 6$. The value $\xi=-1 / 6$ is singled out as only in this case there exists the singular expanding model, which has the asymptotic $\left.a\right|_{t \rightarrow+\infty} \simeq$ const. From three types of the recollapsing models with $\xi<-1 / 6$ only for $\xi=-3 / 2$ we have the following behavior of the scale factor: $\left.a\right|_{t \rightarrow+\infty} \sim e^{-\beta t}$, while for others $\xi$, we get

$$
\begin{aligned}
& \left.a\right|_{t \rightarrow+\infty} \sim t^{(1-\sqrt{6|\xi|) /(3-\sqrt{6|\xi|})}} \text { for }-\frac{3}{2}<\xi<-\frac{1}{6}, \\
& \left.a\right|_{t \rightarrow+t_{0}} \sim\left(t_{0}-t\right)^{(1-\sqrt{6 \mid \xi) /(3-\sqrt{6|\xi|})}} \text { for } \xi<-\frac{3}{2} .
\end{aligned}
$$

The exact general solutions for spatially flat FRW cosmologies with a nonminimally coupled scalar field that has polynomial potentials of the fourth degree $V(\Phi)$ have been obtained in [13]. The main results for the material scalar field are as follows.

(a) For $\xi>0, \Phi^{2}<(\kappa \xi)^{-1 / 2}$, and $V(\Phi)>0$, the solution describes bouncing models of four types with the accelerated expansion at late times by the laws $a \sim e^{H t}$ and $a \sim t^{4 / 3}$. For $\Phi^{2} \neq(\kappa \xi)^{-1 / 2}, V(\Phi)<0$, the qualitative character of the model evolution does not change.

(b) For $\xi<0$, for all $\Phi, V(\Phi)>0$, there are two types of collapsing models and one type of expanding models. For $\xi=-3 / 2$ there exists a unified model of DM and DE. The case with $V(\Phi)<0$ describes the recollapsing models.

The paper is organized as follows. In the next section we present the model and corresponding field equations. In Section 3, we obtain exact general solution of the EinsteinCartan equations for $\xi>0, \Phi^{2}<(\kappa \xi)^{-1 / 2}$. In Section 3.1 we consider the models with a scalar-torsion field and stiff fluid and discuss some interesting particular cases. In Section 3.2 we analyze the mixture of stiff fluid with a scalar-torsion field that has polynomial potentials of the fourth degree. We summarize our results in Section 4. 


\section{Field Equations}

The Lagrangian $€$ of the model is chosen in the form [13]

$$
\mathrm{E}=-\frac{R}{2 \kappa}+\left(\frac{\alpha_{s}}{2}\right)\left[\Phi_{, k} \Phi^{, k}+\xi R \Phi^{2}\right]-V(\Phi)+\mathrm{七}_{\mathrm{fl}},
$$

where $R(\Gamma)$ is the curvature scalar obtained from the full connection $\Gamma_{i j}^{k}=\left\{\begin{array}{l}k \\ i j\end{array}\right\}+S_{i j}^{k}+S_{\cdot i j}^{k}+S_{\cdot j i}^{k} ;\left\{\begin{array}{l}k \\ i j\end{array}\right\}$ are the Christoffel symbols of the second kind; $S_{i j}^{k}=\Gamma_{[i j]}^{k}$ is the torsion tensor; $\kappa=8 \pi G$, where $G$ is the Newtonian constant; $V(\Phi)$ is the potential of a scalar field; $\mathrm{E}_{\mathrm{fl}}$ is the Lagrangian of stiff fluid; $\alpha_{s}=+1$ conforms to the material scalar field; $\alpha_{s}=-1$ corresponds to the ghost scalar field.

The metric $g_{i k}$ has the signature $(-,-,-,+)$; the Riemann and Ricci tensors are defined as

$$
R_{i j k .}^{m}=\Gamma_{j k, i}^{m}-\Gamma_{i k, j}^{m}+\Gamma_{i p}^{m} \Gamma_{j k}^{p}-\Gamma_{j p}^{m} \Gamma_{i k}^{p}
$$

and $R_{j k}=R_{i j k}^{i}$. We should note that in the framework of ECT, a scalar field nonminimally coupled to gravity gives rise to torsion, even though the scalar field has zero spin. It follows from (2) that the torsion can interact with a scalar field only through its trace: $S_{i}=S_{i k}^{k}$. [67]. Hence, the curvature scalar $R(\Gamma)=g^{j k} R_{j k}$ can be presented in the form [67]

$$
R(\Gamma)=R(\{\})+4 \nabla_{k} S^{k}-\left(\frac{8}{3}\right) S_{k} S^{k}
$$

where $R(\{\})$ is the Riemannian part of the curvature built from the Christoffel symbols; $\nabla_{k}$ is the covariant derivative of the Riemannian space.

One can note that the Lagrangian (2) is a covariant generalization of its counterpart in GR, and, for $\xi=1 / 6$, we obtain the so-called conformal coupling (for $V(\Phi)=0$ in the torsionless theory). As shown in [67], when $\alpha_{s}=-1, \xi=$ $-1 / 6, V(\Phi)=-\left(m^{2} / 2\right) \Phi^{2}$, the scalar field corresponding to the Lagrangian (2) is the axion field in GR. In cosmology the axion field is a cold dark matter candidate (see, e.g., $[67,68]$ and references therein).

Varying the action with the Lagrangian (2) in $g_{i j}, S_{k}$, and $\Phi$, we obtain the following set of equations for the gravitational fields and matter:

$$
\begin{gathered}
G_{i j}(\{\})=\kappa\left(T_{i j}^{s}+T_{i j}^{\mathrm{fl}}\right)+\Lambda_{i j}, \\
S^{k}=\frac{3}{2} \xi \Psi \Phi \Phi^{\prime k}, \\
\square \Phi-\xi \Phi R(\Gamma)+\alpha_{s} V^{\prime}=0,
\end{gathered}
$$

where

$$
\begin{gathered}
T_{i j}^{s}=\alpha_{s}\left\{\Phi_{, i} \Phi_{, j}-\frac{1}{2}\left[\Phi_{, m} \Phi^{, m}+\xi R(\{\}) \Phi^{2}-2 \alpha_{s} V(\Phi)\right] g_{i j}\right. \\
+\xi\left[-4 S_{(i} \nabla_{j)}+2 g_{i j} S^{n} \nabla_{n}-\nabla_{i} \nabla_{j}+g_{i j} \square\right. \\
\left.\left.+R_{i j}(\{\})-\Lambda_{i j}\right] \Phi^{2}\right\} \\
T_{i j}^{\mathrm{fl}}=\left(\varepsilon_{\mathrm{fl}}+P_{\mathrm{fl}}\right) u_{i} u_{j}-P_{\mathrm{fl}} g_{i j} \\
\Lambda_{i j}=\frac{8}{3} S_{i} S_{j}-\frac{4}{3} S_{k} S^{k} g_{i j}
\end{gathered}
$$

Here $\varepsilon_{\mathrm{fl}}$ and $P_{\mathrm{fl}}$ are the energy density and pressure of stiff fluid, respectively; $\square$ is the d'Alembertian operator of the Riemannian space; $u_{i}$ is the four-velocity $\left(u_{i} u^{i}=1\right) ; \Psi=$ $\kappa\left(\alpha_{s}-\kappa \xi \Phi^{2}\right)^{-1}$, and $V^{\prime}=\partial V / \partial \Phi$.

It is not difficult to verify that the effective scalar-torsion energy-momentum tensor $T_{i j}^{s(\mathrm{eff})}$,

$$
T_{i j}^{s(\text { eff })}=T_{i j}^{s}+\kappa^{-1} \Lambda_{i j}
$$

and the stiff fluid energy-momentum tensor $T_{i j}^{\mathrm{fl}}$ are separately covariantly conserved since no explicit coupling is assumed between stiff fluid and $\Phi$ :

$$
\nabla^{j} T_{i j}^{s(\mathrm{eff})}=\nabla^{j} T_{i j}^{\mathrm{fl}}=0 .
$$

For spatially flat isotropic and homogeneous models with the metric

$$
d s^{2}=a^{2}(\eta)\left[-d r^{2}-r^{2}\left(d \theta^{2}+\sin ^{2} \theta d \varphi^{2}\right)+d \eta^{2}\right] .
$$

Equations (5) and (7) take the form

$$
\begin{aligned}
& 2 \frac{a^{\prime \prime}}{a}-\frac{a^{\prime 2}}{a^{2}}=\Psi\left[2 \xi \Phi \Phi^{\prime \prime}+2 \xi \frac{a^{\prime}}{a} \Phi \Phi^{\prime}\right. \\
& \left.+\left(-\frac{1}{2}+2 \xi+3 \xi^{2} \Phi^{2} \Psi\right) \Phi^{\prime 2}\right] \\
& +\alpha_{s} a^{2} \Psi\left[V(\Phi)-P_{\mathrm{fl}}\right], \\
& \frac{a^{\prime 2}}{a^{2}}=\Psi\left[\left(\frac{1}{6}-\xi^{2} \Phi^{2} \Psi\right) \Phi^{\prime 2}+2 \xi \frac{a^{\prime}}{a} \Phi \Phi^{\prime}\right] \\
& +\frac{\alpha_{s}}{3} a^{2} \Psi\left[V(\Phi)+\varepsilon_{\mathrm{fl}}\right], \\
& \left(1-6 \xi^{2} \Phi^{2} \Psi\right)\left(\Phi^{\prime \prime}+2 \frac{a^{\prime}}{a} \Phi^{\prime}\right)+6 \xi \frac{a^{\prime \prime}}{a} \Phi \\
& +\alpha_{s} a^{2} V^{\prime}-6 \frac{\alpha_{s}}{\kappa} \xi^{2} \Psi^{2} \Phi \Phi^{\prime 2}=0,
\end{aligned}
$$

where the prime denotes differentiation with respect to $\eta$. Here it is relevant to remark that the use of angular coordinates in metric (13) makes it possible to investigate 
the horizon problem. The passage to the cosmic synchronous time $t$ is fulfilled with the help of the expression

$$
\int a(\eta) d \eta=\int d t
$$

For the stiff fluid we have

$$
P_{\mathrm{fl}}=\varepsilon_{\mathrm{fl}}=C_{\mathrm{fl}} a^{-6},
$$

where $C_{\mathrm{fl}}>0$ is a constant.

Adding (14) and (15) we get

$$
\begin{aligned}
\frac{a^{\prime \prime}}{a}=\Psi[ & \xi \Phi \Phi^{\prime \prime}+2 \xi \frac{a^{\prime}}{a} \Phi \Phi^{\prime}+\frac{2}{3} \alpha_{s} a^{2} V(\Phi) \\
& \left.+\left(-\frac{1}{6}+\xi+\xi^{2} \Phi^{2} \Psi\right) \Phi^{\prime 2}\right]+\frac{\alpha_{s}}{6} a^{2} \Psi\left(\varepsilon_{\mathrm{fl}}-3 P_{\mathrm{fl}}\right) .
\end{aligned}
$$

The substitution of (19) into (16) leads to

$$
\begin{aligned}
\Phi^{\prime \prime} & +2 \frac{a^{\prime}}{a} \Phi^{\prime}-\xi \Phi \Psi \Phi^{\prime 2}+\alpha_{s} a^{2}\left[V^{\prime}+4 \xi \Phi \Psi V(\Phi)\right] \\
& +\alpha_{s} \xi a^{2} \Phi \Psi\left(\varepsilon_{\mathrm{fl}}-3 P_{\mathrm{fl}}\right)=0 .
\end{aligned}
$$

From (20) for stiff fluid and the scalar field potential

$$
V(\Phi)=C_{2}\left(\alpha_{s}-\kappa \xi \Phi^{2}\right)^{2}
$$

we obtain

$$
\left|\Phi^{\prime}\right|\left(\left|\alpha_{s}-\kappa \xi \Phi^{2}\right|\right)^{1 / 2} a^{2}(\eta)=\left(C_{1}+2 \alpha_{s} \kappa \xi \ell C_{\mathrm{fl}} \Phi^{2}\right)^{1 / 2},
$$

where $C_{1}$ and $C_{2}$ are integration constants; $\ell=\operatorname{sgn}\left(\alpha_{s}-\right.$ $\left.\kappa \xi \Phi^{2}\right)$

It must be noted here that the requirement of renormalization of the quantum theory of a scalar field results in the introduction of a nonminimal coupling and the potential in the form of (21) [69].

In this paper, we will restrict our discussion to the material scalar field $\left(\alpha_{s}=+1\right)$. It follows from (22) that there exist solutions for the following restrictions:

$\xi<0$, for all $\Phi$ and $\xi>0, \Phi^{2} \neq B^{2}$. It is not difficult to show that, in terms of physics, reasonable solutions for $a(t)$ and $\Phi(t)$ do not exist for $\xi<0$, for all $\Phi$ and $\xi>0, \Phi^{2}>B^{2}$.

\section{Exact Solution}

The exact general solution to the Einstein-Cartan equations for $\xi>0, \Phi^{2}<B^{2}$ can be presented in the form of quadratures:

$$
\begin{gathered}
a(u)=\beta \cosh u F^{-1 / 2}, \quad \Phi(u)=B \tanh u, \\
\int \frac{\beta d u}{F^{3 / 2} \sqrt{C_{1}+2 C_{\mathrm{fl}} \tanh ^{2} u}}=\int d t, \\
\int \frac{F^{1 / 2} d F}{\sqrt{d_{1} F^{3}+m d_{2}}}=\int \frac{2 n d u}{\cosh u \sqrt{C_{1}+2 C_{\mathrm{f} 1} \tanh ^{2} u}},
\end{gathered}
$$

where

$$
\begin{gathered}
\beta=(\kappa \xi)^{1 / 4}, \quad B=(\kappa \xi)^{-1 / 2}, \quad d_{1}=\frac{\left(C_{1}+2 C_{\mathrm{fl}}\right)}{6 \xi}, \\
d_{2}=\frac{\kappa}{3} C_{2} \beta^{2}, \quad n= \pm 1, \quad m= \begin{cases}+1, & \text { for } V(\Phi)>0, \\
-1, & \text { for } V(\Phi)<0 .\end{cases}
\end{gathered}
$$

An analysis has shown that, depending on the choice of the gravitational field sources, different types of models are possible.

3.1. "Scalar-Torsion Field" + "Stiff Fluid". Let us consider the models without the scalar field potential $V(\Phi)$. It is convenient to discuss separately the cases $C_{1}=0$ and $C_{1} \neq 0$.

3.1.1. Case $\left(C_{1}=0\right)$. From (23), it is possible to express $F=$ $F(u)$ explicitly as

$$
F=D_{1}\left(\left|\tanh \left(\frac{u}{2}\right)\right|\right)^{2 n / \sqrt{6 \xi}}
$$

where $D_{1}>0$ is an integration constant.

For $n=+1$ solution (23) describes bouncing models. The minima of the scale factor,

$$
\begin{gathered}
a_{\min }=\beta D_{1}^{-1 / 2} \cosh u_{\min }\left(\left|\tanh \left(\frac{u_{\min }}{2}\right)\right|\right)^{-1 / \sqrt{6 \xi}}, \\
\Phi_{\min }=B \tan u_{\text {min }},
\end{gathered}
$$

are defined from

$$
\sqrt{6 \xi} \sinh ^{2} u_{\min }=\cosh u_{\min } .
$$

The asymptotic behavior of $a(t)$, when $\left(a-a_{\min }\right) / a_{\min } \ll$ 1 , is

$$
\begin{aligned}
a=a_{\min }\{ & 1+\frac{2 \sqrt{1+24 \xi} C_{\mathrm{fl}} D_{1}^{3}}{\beta^{2}(1+\sqrt{1+24 \xi})} \tanh ^{2} u_{\text {min }} \\
& \left.\times\left(\left|\tanh \left(\frac{u_{\min }}{2}\right)\right|\right)^{6 / \sqrt{6 \xi}} t^{2}\right\} .
\end{aligned}
$$

For $u \in(0, \infty)$ solution (23) has the following asymptotics:

$$
\begin{aligned}
\left.a\right|_{t \rightarrow-\infty} \sim(-t)^{1 / 3}, & \left.\Phi\right|_{t \rightarrow-\infty} \sim(-t)^{-\sqrt{6 \xi} / 3}, \\
\left.a\right|_{t \rightarrow+\infty} & \sim e^{H_{1} t},\left.\quad \Phi\right|_{t \rightarrow+\infty} \simeq B,
\end{aligned}
$$

where $H_{1}=\beta^{-1} D_{1}^{3 / 2}\left(2 C_{\mathrm{fl}}\right)^{1 / 2}$.

It should be observed here that we cannot express $a_{\text {min }}$ and $u_{\min }$ in terms of the present values of the cosmographic parameters $H_{0}, q_{0}$, and $j_{0}$, since three unknown values $\xi, D_{1}$, and $C_{\mathrm{fl}}$ define the Hubble parameter $H_{1}$.

It is easy to see that Hubble's parameter $H_{1}$ can take large values if $\xi \ll 1$. Since the models are considered in 
the framework of a classical theory of gravity, they will be physically admissible provided $\varepsilon<\varepsilon_{\mathrm{pl}}$, where $\varepsilon$ is the total energy density of the scalar-torsion field and stiff fluid and $\varepsilon_{\mathrm{pl}}$ is the Planck energy density. Consequently, the following restriction on $\xi$ is valid for $\xi \ll 1$ :

$$
\xi>36 \kappa^{-3} \varepsilon_{\mathrm{pl}}^{-1} D_{1}^{6} C_{\mathrm{fl}}^{2}
$$

It is interesting to observe that the models (29) are free from the particle horizon; they are nonsingular and admit the late-time accelerating expansion.

For $u \in(-\infty, 0)$ the models with the reverse asymptotics exist:

$$
\begin{gathered}
\left.a\right|_{t \rightarrow-\infty} \sim e^{-H_{1} t},\left.\quad \Phi\right|_{t \rightarrow-\infty} \simeq-B, \\
\left.a\right|_{t \rightarrow+\infty} \sim t^{1 / 3},\left.\quad \Phi\right|_{t \rightarrow+\infty} \sim-t^{-\sqrt{6 \bar{\xi} / 3}} .
\end{gathered}
$$

It follows from (31) that at late times the scalar field disappears with time, and the behavior of the scale factor corresponds to the decelerated expansion.

It is easy to verify that models (29) and (31) are regular due to the violation of the strong energy condition with a scalar-torsion field. It is not difficult to show that, for minima of the scale factor and for the de Sitter-like asymptotics, the contribution of the scalar-torsion field dominates, while for $a \sim t^{1 / 3}$ the contribution of the stiff fluid dominates.

As pointed out in [70,71], since the current torsion is extremely small, only those solutions should be considered as physical solutions towards current epoch, in which torsion disappears with time. In this connection, it should be noted that the square of the trace of torsion $S^{2}=S_{k} S^{k}$ has the following asymptotics:

(1) stiff fluid dominated era

$$
\left.S^{2}\right|_{t \rightarrow \mp \infty} \sim(\mp t)^{-2(3+2 \sqrt{6 \xi}) / 3} \longrightarrow 0,
$$

(2) de Sitter regime

$$
\left.S^{2}\right|_{t \rightarrow \mp \infty} \simeq\left(\frac{9}{4}\right) H_{1}^{2}=\text { const. }
$$

It follows from (32) that for the stiff fluid dominated era the torsion disappears with time. The formula (33) shows that for the de Sitter regime the torsion asymptotically tends to a finite value. For the current epoch, provided that $H_{1}=H_{0}$, where $H_{0}$ is the present-day value of the Hubble's parameter, we have the following in natural units $(\hbar=c=1)$ :

$$
\left.S^{2}\right|_{t \rightarrow+\infty} \sim 10^{-84} \mathrm{GeV}^{2} \text {. }
$$

That is, the current torsion is very small.

For minima of the scale factor the square of the trace of torsion is

$$
S^{2}=\left(\frac{9}{4}\right) H_{1}^{2} \tanh ^{4} u_{\min }\left(\left|\tanh \left(\frac{u_{\min }}{2}\right)\right|\right)^{6 / \sqrt{6 \xi}}=\text { const. }
$$

That is, the torsion is finite.
It must be noted that, for $\sqrt{6 \xi}=3$, the solution may be written in a parametric form

$$
\begin{gathered}
a(t)=\beta D_{1}^{-1 / 2} x^{-1 / 3} \sqrt{1+x^{2}}\left(1+\sqrt{1+x^{2}}\right)^{1 / 3}, \\
\Phi(t)=B \frac{n_{1} x}{\sqrt{1+x^{2}}}, \\
S^{2}(t)=\frac{9 H_{1}^{2} x^{6}}{4\left(1+x^{2}\right)^{2}\left(1+\sqrt{1+x^{2}}\right)^{2}}, \\
\ln \left|x+\sqrt{1+x^{2}}\right|-x^{-1}\left(1+\sqrt{1+x^{2}}\right)=n_{1} H_{1} t
\end{gathered}
$$

where $x \in(0, \infty), n_{1}=+1$ for $u \in(0, \infty)$ and $n_{1}=-1$ for $u \in(-\infty, 0)$.

For $n=-1, u \in(0, \infty)$, solution (23) corresponds to the singular expanding models with de Sitter asymptotics for the late times:

$$
\begin{gathered}
\left.a\right|_{t \rightarrow-t_{0}} \sim\left(t_{0}+t\right)^{1 / 3},\left.\quad \Phi\right|_{t \rightarrow-t_{0}} \sim\left(t_{0}+t\right)^{\sqrt{6 \xi / 3}}, \\
\left.a\right|_{t \rightarrow+\infty} \sim e^{H_{1} t},\left.\quad \Phi\right|_{t \rightarrow+\infty} \simeq B,
\end{gathered}
$$

while for $u \in(-\infty, 0)$, this solution conforms to the models with the reverse asymptotics, which describe the collapsing models.

It is easy to verify that for $t \rightarrow-t_{0}$ the contribution of the stiff fluid dominates. The behavior of $S^{2}$ for $t \rightarrow+\infty$ is described by the formula (33). It is interesting to observe that the square of the trace of torsion has the following asymptotics for $t \rightarrow-t_{0}$ :

$$
\left.S^{2}\right|_{t \rightarrow-t_{0}} \sim\left(t_{0}+t\right)^{2(2 \sqrt{6 \xi}-3) / 3}= \begin{cases}\rightarrow \infty, & \text { for } \sqrt{6 \xi}<\frac{3}{2}, \\ =\text { const, } & \text { for } \sqrt{6 \xi}=\frac{3}{2} \\ \rightarrow 0, & \text { for } \sqrt{6 \xi}>\frac{3}{2}\end{cases}
$$

That is, there is the specific value of the coupling constant: $\xi=3 / 8$.

Thus, for the Big Bang singularity of the form $\left.a\right|_{t \rightarrow-t_{0}} \sim$ $\left(t_{0}+t\right)^{1 / 3}: a \rightarrow \infty, \varepsilon \rightarrow \infty, P \rightarrow \infty$, where $P$ is the total pressure of the scalar-torsion field and stiff fluid, the torsion can be singular for $\sqrt{6 \xi}<3 / 2$, constant for $\sqrt{6 \xi}=3 / 2$, or tend to zero for $\sqrt{6 \xi}>3 / 2$. The solutions for the three types of $S^{2}$ behavior can be presented in a parametric form $(\Phi(u)=$ $B \tanh u$ ): 
(1) $\sqrt{6 \xi}=3 / 4$,

$$
\begin{aligned}
& a(u)=\beta D_{1}^{-1 / 2} \cosh u \tanh ^{4 / 3}\left(\frac{u}{2}\right), \\
& S^{2}(u)=\left(\frac{9}{4}\right) H_{1}^{2} \tanh ^{4} u \tanh ^{-8}\left(\frac{u}{2}\right), \\
& \left(5+\tanh ^{2}\left(\frac{u}{2}\right)\right)\left(2 \cosh ^{2}\left(\frac{u}{2}\right)\right)^{-1} \\
& \quad+4 \ln \left(\cosh \left(\frac{u}{2}\right)\right)=2 H_{1}\left(t_{0}+t\right),
\end{aligned}
$$

where $t_{0}=5\left(4 H_{1}\right)^{-1}$.

(2) $\sqrt{6 \xi}=3 / 2$,

$$
\begin{gathered}
a(u)=\beta D_{1}^{-1 / 2} \cosh u \tanh ^{2 / 3}\left(\frac{u}{2}\right), \\
S^{2}(u)=\left(\frac{9}{4}\right) H_{1}^{2} \tanh ^{4} u \tanh ^{-4}\left(\frac{u}{2}\right), \\
\cosh ^{-2}\left(\frac{u}{2}\right)+4 \ln \left(\cosh \left(\frac{u}{2}\right)\right)=2 H_{1}\left(t_{0}+t\right),
\end{gathered}
$$

where $t_{0}=\left(2 H_{1}\right)^{-1}$.

(3) $\sqrt{6 \xi}=3$,

$$
\begin{gathered}
a(u)=\beta D_{1}^{-1 / 2} \cosh u\left(\left|\tanh \left(\frac{u}{2}\right)\right|\right)^{1 / 3}, \\
S^{2}(u)=\left(\frac{9}{4}\right) H_{1}^{2} \tanh ^{4} u \tanh ^{-2}\left(\frac{u}{2}\right), \\
u-\tanh \left(\frac{u}{2}\right)=2 H_{1}\left(t_{0}+t\right),
\end{gathered}
$$

where $t_{0}=0$.

3.1.2. Case $\left(C_{1} \neq 0\right)$. An analysis has shown that for $C_{1}>0$ the solution exists only for $n=-1$ and describes nonsingular expanding models with the asymptotics:

$$
\begin{gathered}
\left.a\right|_{t \rightarrow 0} \simeq a_{01}\left(1+\beta^{-1} D_{1}^{3 / 2} \sqrt{\frac{C_{1}+2 C_{\mathrm{fl}}}{6 \xi}} t\right), \\
\left.\Phi\right|_{t \rightarrow 0} \sim t,\left.\quad a\right|_{t \rightarrow+\infty} \sim e^{H_{2} t},\left.\quad \Phi\right|_{t \rightarrow+\infty} \simeq B,
\end{gathered}
$$

where $a_{01}=a(t=0)=\beta D_{1}^{-1 / 2}, H_{2}=\beta^{-1} D_{1}^{3 / 2}\left(C_{1}+2 C_{\mathrm{fl}}\right)^{1 / 2}$.

It is not difficult to show that for $C_{1}<0$, we have the same qualitative picture of the evolution of models as for $C_{1}>0$.

3.2. "Scalar-Torsion Field" + "V( $\Phi) "+$ "Stiff Fluid". Let us consider the models for which all sources of gravitational field from the Lagrangian (2) are taken into account.

3.2.1. Case $\left(C_{1}=0\right)$. From (23) we find the expression for $F(u)$,

$$
F^{3 / 2}=\left(W^{2}-m d_{2}\right)\left(2 d_{1}^{1 / 2} W\right)^{-1},
$$

where $W=D_{2}(|\tanh (u / 2)|)^{3 n / \sqrt{6 \xi}} ; D_{2}>0$ is an integration constant.

An analysis has shown that this solution with $V(\Phi)<0$, for all $n$ and $V(\Phi)>0, n=-1$, and $D_{2}^{2}>d_{2}$ for $u \in$ $(0, \infty)$ describes singular expanding models with the de Sitter asymptotic at late times:

$$
\begin{aligned}
& \left.a\right|_{t \rightarrow 0} \sim t^{1 / 3},\left.\quad \Phi\right|_{t \rightarrow 0} \sim t^{\sqrt{6 \xi} / 3},\left.\quad S^{2}\right|_{t \rightarrow 0} \sim t^{2(2 \sqrt{6 \xi}-3) / 3}, \\
& \left.a\right|_{t \rightarrow \infty} \sim e^{H_{3} t},\left.\quad \Phi\right|_{t \rightarrow \infty} \simeq B,\left.\quad S^{2}\right|_{t \rightarrow \infty} \simeq\left(\frac{9}{4}\right) H_{3}^{2},
\end{aligned}
$$

where $H_{3}=(6 \xi)^{1 / 2}\left(2 \beta D_{2}\right)^{-1}\left(D_{2}^{2}-m d_{2}\right)$. Note that for $u \in$ $(-\infty, 0)$ the reverse asymptotic behavior takes place.

For $V(\Phi)<0, n=+1, D_{2}^{2}=d_{2}$, and $\sqrt{6 \xi}=3$, the solution can be expressed in elementary functions:

$$
\begin{gathered}
a(t)=A_{1} \cosh \gamma t(|\tanh \gamma t|)^{1 / 3}, \quad \Phi(t)=B \tanh \gamma t, \\
S^{2}(t)=\left(\frac{9}{4}\right) H_{3}^{2} \tanh ^{2} \gamma t,
\end{gathered}
$$

where $A_{1}=\beta\left(3 D_{2}\right)^{-1 / 3}\left(2 C_{\mathrm{fl}}\right)^{1 / 6}, \gamma=3 \beta^{-1} D_{2}$.

It should be pointed out that, for $t \in(0, \infty)$, we have the expanding models, while, for $t \in(-\infty, 0)$, we get the collapsing models.

For $V(\Phi)>0, n=-1, D_{2}^{2}=d_{2}$, and $u \in(0, \infty)$, this solution corresponds to the singular expanding models with the power-law asymptotic at late times:

$$
\begin{gathered}
\left.a\right|_{t \rightarrow 0} \sim t^{1 / 3},\left.\quad \Phi\right|_{t \rightarrow 0} \sim t^{\sqrt{6 \xi} / 3}, \\
\left.S^{2}\right|_{t \rightarrow 0} \sim t^{2(2 \sqrt{6 \xi}-3) / 3}, \\
\left.a\right|_{t \rightarrow+\infty} \sim t^{4 / 3},\left.\quad \Phi\right|_{t \rightarrow+\infty} \simeq B, \\
\left.S^{2}\right|_{t \rightarrow+\infty} \sim t^{-2} \longrightarrow 0 .
\end{gathered}
$$

It is easy to see that the case for $u \in(-\infty, 0)$ conforms to the models with the reverse asymptotics.

It should be observed here that the accelerated expansion of models with the power-law asymptotic at late stages of the cosmological evolution has its origin in "Scalar-torsion field" + " $V(\Phi)$."

For $\sqrt{6 \xi}=3$, the solution can be presented in elementary functions:

$$
\begin{gathered}
a(t)=A_{2}(|t|)^{1 / 3}\left(1+\gamma^{2} t^{2}\right)^{1 / 2}, \quad \Phi(t)=\frac{\gamma B t}{\sqrt{1+\gamma^{2} t^{2}}}, \\
S^{2}(t)=\frac{9 \gamma^{4} t^{2}}{4\left(1+\gamma^{2} t^{2}\right)^{2}},
\end{gathered}
$$

where $A_{2}=\beta^{2 / 3}\left(2 C_{\mathrm{fl}}\right)^{1 / 6}$. It follows from (47) that for $t \rightarrow 0$ the square of the trace of torsion has the following behavior: $\left.S^{2}\right|_{t \rightarrow 0} \sim t^{2} \rightarrow 0$. 

$3 / 2$,

The analytic solution in elementary functions for $\sqrt{6 \xi}=$

$$
\begin{gathered}
a(t)=A_{3}\{|t|(1+\gamma|t|)\}^{1 / 3}(1+2 \gamma|t|)^{2 / 3}, \\
\Phi(t)=2 B\{\gamma|t|(1+\gamma|t|)\}^{1 / 2}(1+2 \gamma|t|)^{-1}, \\
S^{2}(t)=54 \xi \beta^{-2} D_{2}^{2}(1+2 \gamma|t|)^{-2},
\end{gathered}
$$

where $A_{3}=\beta^{2 / 3}\left(8 C_{\mathrm{fl}}\right)^{1 / 6}$, corresponds to the following behavior of $S^{2}$ for $t=0: S^{2}=54 \xi \beta^{-2} D_{2}^{2}=$ const.

Formulae (47) and (48) show that the analytic representation of the solution depends on the behavior of the square of the trace of torsion at early times.

The solution in a parametric form for $\sqrt{6 \xi}=3 / 4$,

$$
\begin{aligned}
& a(u)= 2 \beta\left(\frac{2 C_{\mathrm{fl}}}{9 d_{2}}\right)^{1 / 6} \cosh u \tanh ^{4 / 3}\left(\frac{u}{2}\right) \\
& \times\left(1-\tanh ^{8}\left(\frac{u}{2}\right)\right)^{-1 / 3}, \\
& S^{2}(u)=\left(\frac{81 d_{2}}{64}\right)\left(\frac{2}{3 \kappa}\right)^{1 / 2} \tanh ^{4} u \tanh ^{-8} \\
& \times\left(\frac{u}{2}\right)\left(1-\tanh ^{8}\left(\frac{u}{2}\right)\right)^{2}, \\
& \Phi(u)= B \tanh u, \\
& \cosh ^{2}\left(\frac{u}{2}\right)-\arctan \left(\tanh ^{2}\left(\frac{u}{2}\right)\right)=1+2 \gamma|t|,
\end{aligned}
$$

conforms to the following behavior of $S^{2}$ for $t \rightarrow 0$ : $\left.S^{2}\right|_{t \rightarrow 0} \rightarrow \infty$.

For $V(\Phi)>0, n=+1$, solution (23) exists for $u \in$ $(-\infty,-D]$ and $u \in[D,+\infty)$, where $D$ is a constant. With $u \in[D,+\infty)$ provided that

$$
\lambda=D_{2}\left(\left|\tanh \left(\frac{D}{2}\right)\right|\right)^{3 / \sqrt{6 \xi}}, \quad \lambda^{2}=g_{1} d_{2}, \quad g_{1}>1,
$$

the evolution of models for $t \rightarrow \infty$ is identical to the models (44), while for $t \rightarrow 0$ we have

(a) $\left(g_{1}-1\right)(6 \xi)^{1 / 2} \sinh ^{2} D>\left(g_{1}+1\right) \cosh D$,

$$
\begin{aligned}
\left.a\right|_{t \rightarrow 0} & \simeq a_{02} \\
& \times\left[1+\frac{\left(g_{1}-1\right)(6 \xi)^{1 / 2} \sinh ^{2} D-\left(g_{1}+1\right) \cosh D}{2 \beta g_{1}^{1 / 2} \cosh ^{2} D} d_{2}^{1 / 2} t\right],
\end{aligned}
$$

where $a_{02}=a(t=0)=\beta\left(g_{1}-1\right)^{-1 / 3}\left(8 g_{1} C_{\mathrm{fl}} / 6 \xi d_{2}\right)^{1 / 6} \cosh D$,

(b) $\left(g_{1}-1\right)(6 \xi)^{1 / 2} \sinh ^{2} D=\left(g_{1}+1\right) \cosh D$,

$$
\left.a\right|_{t \rightarrow 0} \simeq a_{02}\left[1+d_{2} \chi_{1}\left(8 g_{1} \beta^{2} \cosh ^{2} D\right)^{-1} t^{2}\right],
$$

where $\chi_{1}=\left(g_{1}-1\right)\left[\left(g_{1}+1\right) \cosh D-\left(g_{1}-1\right)\right](6 \xi)^{1 / 2}+11 g_{1}^{2}-$ $2 g_{1}-1$.

For (51) and (52), the expressions for $\Phi$ and $S^{2}$ have the form

$$
\Phi(t=0)=B \tanh D, \quad S^{2}(t=0)=\frac{27 \xi d_{2}\left(g_{1}-1\right)^{2}}{8 g_{1} \beta^{2}} .
$$

It is easy to see that for $g_{1}>1$ the solution describes the expanding nonsingular models. Here it is relevant to remark that the accelerated expansion both at early stages and at late stages of the cosmological evolution is characteristic for case (b).

It is not difficult to verify that for $u \in(-\infty,-D]$ we get the models with the reverse asymptotics.

For $V(\Phi)>0, n=+1$, and $g_{1}=1$, solution (23) describes bouncing models with the asymptotics

$$
\begin{gathered}
\left.a\right|_{t \rightarrow-\infty} \sim e^{-H_{4} t},\left.\quad \Phi\right|_{t \rightarrow-\infty} \simeq B \tanh D, \\
\left.S^{2}\right|_{t \rightarrow-\infty} \sim e^{6 H_{4} t} \longrightarrow 0, \\
\left.a\right|_{t \rightarrow+\infty} \sim e^{H_{3} t},\left.\quad \Phi\right|_{t \rightarrow+\infty} \simeq B, \\
\left.S^{2}\right|_{t \rightarrow+\infty} \simeq\left(\frac{9}{4}\right) H_{3}^{2}=\text { const },
\end{gathered}
$$

where $H_{4}=\lambda(\beta \cosh D)^{-1}$.

The minima of the scale factor,

$$
\begin{gathered}
a_{\min }=\beta\left(g_{2}-1\right)^{-1 / 3}\left(\frac{4 g_{2} C_{\mathrm{fl}}}{3 \xi d_{2}}\right)^{1 / 6} \cosh u_{\min }, \\
\Phi_{\min }=B \tanh u_{\min }
\end{gathered}
$$

where $g_{2}=\left(\left|\tanh \left(u_{\min } / 2\right) / \tanh (D / 2)\right|\right)^{6 / \sqrt{6 \xi}}>1$, are defined from

$$
\left(g_{2}-1\right)(6 \xi)^{1 / 2} \sinh ^{2} u_{\min }=\left(g_{2}+1\right) \cosh u_{\text {min }} .
$$

The asymptotic behavior of $a(t)$, when $\left(a-a_{\min }\right) / a_{\min } \ll$ 1 , is

$$
a=a_{\min }\left[1+d_{2} \chi_{2}\left(8 g_{2} \beta^{2} \cosh ^{3} u_{\min }\right)^{-2} t^{2}\right],
$$

where

$$
\begin{aligned}
& \chi_{2}= {\left[12 g_{2}^{2}-(6 \xi)^{1 / 2}\left(g_{2}+1\right)^{2}\right] \cosh u_{\text {min }} } \\
&+(6 \xi)^{1 / 2}\left(g_{2}^{2}-1\right), \\
& 12 g_{2}^{2}>(6 \xi)^{1 / 2}\left(g_{2}+1\right)^{2} .
\end{aligned}
$$

The value of $S^{2}$ for minima of the scale factor is

$$
S_{\min }^{2}=9 d_{2}\left(g_{2}+1\right)^{2}\left(16 g_{2} \beta^{2}\right)^{-1} \cosh ^{-2} u_{\min } .
$$

That is, the torsion is finite. 
3.2.2. Case $\left(C_{1} \neq 0\right)$. An analysis has shown that with $C_{1} \neq 0$ for all $V(\Phi)$ only the nonsingular expanding models of types (42) are possible.

\section{Conclusion}

In this paper, exact general solution for spatially flat isotropic and homogeneous cosmological models in ECT with stiff fluid and a nonminimally coupled material scalar field with a potential $V(\Phi)$ has been obtained and analyzed for an arbitrary positive coupling constant $\xi$.

In order to elucidate the role of the stiff fluid in the Einstein-Cartan cosmology, we should carry out a comparative analysis of the corresponding cosmological models obtained in this paper and those in [12] for "Scalar-torsion field" and in [13] for "Scalar-torsion field" + "V( $\Phi)$."

By comparing these models, we arrive at the conclusion that for the cosmological models with "Scalar-torsion field" + "Stiff fluid" and "Scalar-torsion field" + "V $(\Phi)$ " + "Stiff fluid" the presence of stiff fluid leads to

(i) integrability of the Einstein-Cartan equations for $\xi>$ $0, \Phi^{2}<B^{2}$ only,

(ii) the existence of singular expanding models with the power-law $\left(a \sim t^{1 / 3}\right)$ evolution at early times and de Sitter evolution at late times,

(iii) the existence of nonsingular (not bouncing) expanding models with de Sitter asymptotic at late times,

(iv) the existence of the specific value of the coupling constant $\xi: \xi=3 / 8$.

Furthermore, for the models with the "Scalar-torsion field" + "Stiff fluid" the presence of stiff fluid leads to the existence of asymmetrically bouncing models with the asymptotics $\left.a\right|_{t \rightarrow-\infty} \sim(-t)^{1 / 3}$ and $\left.a\right|_{t \rightarrow+\infty} \sim e^{H_{1} t}$. These bouncing models are of interest from the viewpoint of modern cosmology: these models are free from the particle horizon, they are nonsingular, they admit the late-time accelerating expansion, and the current torsion for them is very small.

Additionally, stiff fluid in the mixture with the "Scalartorsion field" + " $V(\Phi)$ " results in a decreased number of types of bouncing models and the existence of singular expanding models with the power-law $\left(a \sim t^{1 / 3}\right)$ evolution at early times and with the power-law $\left(a \sim t^{4 / 3}\right)$ evolution at late times.

It should be observed that the existence of the powerlaw $\left(a \sim t^{1 / 3}\right)$ evolution of the scale factor in the presence of stiff fluid is expected but not necessary. For example, for flat Friedmann universes filled by radiation, stiff fluid, and a nonminimally coupled ghost scalar field with polynomial potentials of the fourth degree [66], there is not such asymptotic for the scale factor, since the "kinetic term" of the scalar-torsion field compensates for the energy density of stiff fluid.

Comparing the models obtained in this work with the DE models that could admit similar behaviors we note the following. The behavior of the scale factor by the law $a \sim e^{H t}$ at late times corresponds to $\Lambda$ CDM model, while the behavior like $a \sim t^{4 / 3}$ at late times conforms to a quintessence model of DE [10, 72].

\section{Acknowledgments}

The author is grateful to the unknown reviewers for the useful remarks.

\section{References}

[1] A. G. Riess, L.-G. Sirolger, J. Tonry et al., “Type Ia supernova discoveries at $z>1$ from the hubble space telescope: evidence for past deceleration and constraints on dark energy evolution," The Astrophysical Journal, vol. 607, article 665, 2004.

[2] E. Komatsu, J. Dunkley, M. R. Nolta et al., "Five-year Wilkinson microwave anisotropy probe observations: cosmological interpretation," The Astrophysical Journal Supplement Series, vol. 180, no. 2, article 330, 2009.

[3] W. M. Wood-Vasey, G. Miknaitis, C. W. Stubbs et al., "Observational constraints on the nature of dark energy: first cosmological results from the essence supernova survey," Astrophysical Journal Letters, vol. 666, no. 2, pp. 694-715, 2007.

[4] M. Tegmark, M. R. Blanton, M. A. Strauss et al., "The threedimensional power spectrum of galaxies from the sloan digital sky survey," Astrophysical Journal Letters, vol. 606, no. 2, pp. 702-740, 2004.

[5] P. Astier, J. Guy, N. Regnault et al., "The supernova legacy survey: measurement of $\Omega_{\mathrm{M}}, \Omega_{\wedge}$ and w from the first year data set," Astronomy \& Astrophysics, vol. 447, no. 1, pp. 31-48, 2006.

[6] A. H. Jaffe, P. A. R. Ade, A. Balbi et al., "Cosmology from MAXIMA-1, BOOMERANG, and COBE DMR cosmic microwave background observations," Physical Review Letters, vol. 86, no. 16, pp. 3475-3479, 2001.

[7] T. Padmanabham, "Cosmological constant—the weight of the vacuum," Physics Reports, vol. 380, no. 5-6, pp. 335-320, 2003.

[8] P. J. E. Peebles and B. Ratra, "The cosmological constant and dark energy," Reviews of Modern Physics, vol. 75, no. 2, pp. 599606, 2003.

[9] V. Sahni and A. Starobinsky, "Reconstructing dark energy," International Journal of Modern Physics D, vol. 15, no. 12, pp. 2105-2132, 2006.

[10] M. Li, X.-D. Li, S. Wang, and Y. Wand, “Dark energy," Communications in Theoretical Physics, vol. 56, no. 3, article 525, 2011.

[11] A. M. Galiakhmetov, "Exact anisotropic scalar field cosmologies in Einstein-Cartan theory," Gravitation and Cosmology, vol. 13, pp. 217-223, 2007.

[12] A. M. Galiakhmetov, "Exact isotropic scalar field cosmologies in Einstein-Cartan theory," Classical and Quantum Gravity, vol. 27, no. 5, Article ID 055008, 2010.

[13] A. M. Galiakhmetov, "Cosmology with polynomial potentials of the fourth degree in Einstein-Cartan theory," Classical and Quantum Gravity, vol. 28, no. 10, Article ID 105013, 2011.

[14] É.. Cartan, "Sur les espaces à connexion affine et la théorie de la relativité généralisée, partie I," Annales Scientifiques de l'École Normale Supérieure, vol. 40, supplement, p. 325, 1923.

[15] E. Cartan, "Sur les varietes à connexion et la theorie de la relativité généralisée (suite)," Annales Scientifiques de l'École Normale Supérieure, vol. 41, supplement, pp. 1-25, 1924. 
[16] E. Cartan, "Sur les espaces à connexion affine et la théorie de la relativité généralisée partie II," Annales Scientifiques de l’École Normale Supérieure, vol. 42, supplement, pp. 17-88, 1925.

[17] F. W. Hehl and Y. N. Obukhov, "Elie Cartan's torsion in geometry and in field theory, an essay," Annales de la Fondation Louis de Broglie, vol. 32, p. 157, 2007.

[18] F. W. Hehl, P. Von Der Heyde, G. D. Kerlick, and J. M. Nester, "General relativity with spin and torsion: foundations and prospects," Reviews of Modern Physics, vol. 48, no. 3, pp. 393416, 1976.

[19] V. N. Ponomarev, A. O. Barvinsky, and N. Yu. Obuhov, Geometrodynamics Methods and Gauge Approach in the Theory of Gravity, Energoatomizdat, Moscow, Russia, 1985.

[20] A. V. Minkevich and A. S. Garkun, "Analysis of inflationary cosmological models in gauge theories of gravitation," Classical and Quantum Gravity, vol. 23, no. 12, article 4237, 2006.

[21] A. Trautman, "Einstein-Cartan theory," in Encyclopedia of Mathematical Physics, J. P. Françoise, G.-L. Naber, and S. T. Tsou, Eds., pp. 189-195, Elsevier, Oxford, UK, 2006.

[22] P. Baekler and F. W. Hehl, "Beyond Einstein-Cartan gravity: quadratic torsion and curvature invariants with even and odd parity including all boundary terms," Classical and Quantum Gravity, vol. 28, no. 21, Article ID 215017, 2011.

[23] W. A. Kopczynsky, "A non-singular universe with torsion," Physics Letters A, vol. 39, no. 3, pp. 219-220, 1972.

[24] J. Tafel, "Cosmological models with a spinor field," Bulletin de L'Académie Polonaise des Sciences, Série des Sciences Mathématiques, Astronomiques et Physiques, vol. 25, p. 593, 1977.

[25] A. Teixeira, "Homogeneous, nonsingular, closed EinsteinCartan cosmological model," Physical Review D, vol. 31, pp. 2132-2134, 1985.

[26] M. Gasperini, "Repulsive gravity in the very early Universe," General Relativity and Gravitation, vol. 30, pp. 1703-1709, 1998.

[27] V. A. Korotkii and Y. N. Obukhov, "Rotating and expanding cosmology in ECSK-theory," Astrophysics and Space Science, vol. 198, no. 1, pp. 1-12, 1992.

[28] M. Poplawski, "Cosmology with torsion: an alternative to cosmic inflation," Physics Letters B, vol. 694, no. 3, pp. 181-185, 2010.

[29] H. Rumph, "Particle creation by torsion and electromagnetic fields," General Relativity and Gravitation, vol. 10, p. 456, 1979.

[30] M. Seitz, "On solutions of the Einstein-Cartan-Dirac theory," Classical and Quantum Gravity, vol. 2, article 919, no. 6, p. 919, 1985.

[31] D. D. Ivanenko, P. I. Pronin, and G. A. Sardanashvili, Gauge Theory of Gravity, Moscow State University Press, Moscow, Russia, 1985.

[32] V. Krechet, Problems of gravitational interaction of the phisical fields in an affine connected spaces [PhD thesis], Yaroslavl State Pedagogical University, 1984.

[33] M. A. J. Vandyck, "On the motion of test particles in a plane wave of supergravity," Classical and Quantum Gravity, vol. 4, no. 3, article 683, 1987.

[34] S. D. Odintsov, "The nonsingular quantum cosmological models with nontrivial topology in conformal (Super) gravity," Europhysics Letters, vol. 8, no. 4, article 309, 1989.

[35] I. L. Buchbinder and S. D. Odintsov, "The behaviour of effective coupling constants in "Finite" grand unification theories in curved space-time with torsion," Europhysics Letters, vol. 8, no. 7, article 595, 1989.
[36] M. W. Kalinowski, "On a generalization of the Einstein-Cartan theory and the Kaluza-Klein theory," Letters in Mathematical Physics, vol. 5, p. 489, 1958.

[37] G. German, "On Kaluza-Klein theory with torsion," Classical and Quantum Gravity, vol. 2, no. 4, 455, 1982.

[38] S. Yu. Vladimirov and A. D. Popov, "Compound torsion and spontaneous compactification in Kaluza-Klein theories," Vestnik Moskovskogo Universiteta, Fizika, Astronomiya, vol. 29, no. 4, pp. 28-32, 1988.

[39] K. Akdeniz, A. Kizilersu, and E. Rizaoglu, "Instanton and eigenmodes in a two-dimensional theory of gravity with torsion," Physics Letters B, vol. 215, no. 1, pp. 81-83, 1988.

[40] P. Kuusk, "The heterotic string and the geometry of the supersymmetric Einstein-Yang-Mills background," General Relativity and Gravitation, vol. 21, no. 2, pp. 185-200, 1989.

[41] W. M. Baker, "Cosmic strings in Riemann-Cartan spacetimes," Classical and Quantum Gravity, vol. 7, no. 5, article 717, 1990.

[42] S. Capozziello, R. Cianci, C. Stornaiolo, and S. Vignolo, "F(R) gravity with torsion: the metric-affine approach," Classical and Quantum Gravity, vol. 24, no. 24, pp. 6417-6430, 2007.

[43] S. Capozziello, R. Cianci, C. Stornaiolo, and S. Vignolo, " $f(R)$ Cosmology with torsion," Physica Scripta, vol. 78, no. 6, Article ID 0605010, 2008.

[44] S. Capozziello and S. Vignolo, "Metric-affine $f(R)$-gravity with torsion: an overview, Annalen der Physik, vol. 19, no. 3-5, pp. 238-248, 2010.

[45] L. Fabbri and S. Vignolo, "Dirac fields in $f(R)$-gravity with torsion," Classical and Quantum Gravity, vol. 28, no. 12, Article ID 125002, 2011.

[46] G. R. Bengochea and R. Ferraro, "Dark torsion as the cosmic speed-up," Physical Review D, vol. 79, no. 12, Article ID 124019, 5 pages, 2009.

[47] E. V. Linder, "Einstein's other gravity and the acceleration of the Universe," Physical Review D, vol. 81, no. 12, Article ID 127301, 2010.

[48] K. Bamba, C. Q. Geng, C. C. Lee, and L. W. Luo, "Equation of state for dark energy in $f(T)$ gravity," Journal of Cosmology and Astroparticle Physics, vol. 2011, article 021, 2011.

[49] K. Hayashi and T. Shirafuji, "New general relativity," Physical Review D, vol. 19, no. 12, pp. 3524-3553, 1979.

[50] S. Carloni, S. Capozziello, J. A. Leach, and P. K. S. Dunsby, "Cosmological dynamics of scalar-tensor gravity," Classical and Quantum Gravity, vol. 25, no. 3, Article ID 035008, 2008.

[51] F. L. Bezrukov and M. Shaposhnikov, "The standard model Higgs boson as the inflaton," Physics Letters B, vol. 659, no. 3, pp. 703-706, 2008.

[52] K. Nozari and S. D. Sadatian, "Non-minimal inflation after WMAP3," Modern Physics Letters A, vol. 23, no. 34, article 2933, 2008.

[53] A. de Simone, M. P. Hertzberg, and F. Wilczek, "Running inflation in the standard model," Physics Letters B, vol. 678, no. 1, pp. 1-8, 2009.

[54] A. O. Barvinsky, A. Y. Kamenshchik, C. Kiefer, A. A. Starobinsky, and C. Steinwachs, "Asymptotic freedom in inflationary cosmology with a non-minimally coupled Higgs field," Journal of Cosmology and Astroparticle Physics, vol. 2009, article 003, 2009.

[55] R. Gannouji, D. Polarski, A. Ranquet, and A. A. Starobinsky, "Scalar-tensor models of normal and phantom dark energy," Journal of Cosmology and Astroparticle Physics, vol. 2006, article 016, 2006. 
[56] M. Szydlowsky and O. Hrycyna, "Scalar field cosmology in the energy phase-space-unified description of dynamics," Journal of Cosmology and Astroparticle Physics, vol. 2009, article 039, 2009.

[57] A. Yu. Kamenshchik, A. Tronconi, and G. Venturi, "Dynamical dark energy and spontaneously generated gravity," Physics Letters B, vol. 713, no. 4-5, pp. 358-364, 2012.

[58] P. J. E. Peebles and B. Ratra, "Cosmology with a time-variable cosmological 'constant"' Astrophysical Journal, vol. 325, pp. L17L20, 1988 .

[59] J. Garcia-Bellido and A. Linde, "Stationarity of inflation and predictions of quantum cosmology," Physical Review D, vol. 51, no. 2, pp. 429-443, 1995.

[60] D. La and P. J. Steinhardt, "Extended inflationary cosmology," Physical Review Letters, vol. 62, no. 4, pp. 376-378, 1989.

[61] K. P. Staniukovich and V. N. Melnikov, Hydrodynamics, Fields and Constants in Gravitation Theory, Energoatomizdat, Moscow, Russia, 1983.

[62] A. de la Maccora, "Realistic particle physics dark energy model," Physical Review D, vol. 72, no. 4, Article ID 043508, 7 pages, 2005.

[63] A. Linde, Particle Physics and Inflationary Cosmology, Gordon and Breach, New York, NY, USA, 1990.

[64] G. Felder, A. Frolov, L. Kofman, and A. Linde, "Cosmology with negative potentials," Physical Review D, vol. 66, no. 2, Article ID 023507, 26 pages, 2002.

[65] M. Cataldo and L. P. Chimento, "Crossing the phantom divide with a classical Dirac field," Astrophysics and Space Science, vol. 333, no. 1, pp. 277-285, 2011.

[66] A. M. Galiakhmetov, "Multi-component Einstein-Cartan cosmologies," International Journal of Theoretical Physics, vol. 52, no. 3, pp. 765-778, 2013.

[67] V. G. Krechet and D. V. Sadovnikov, "Cosmology in an affinemetric theory of gravity with a scalar field," Gravitation and Cosmology, vol. 3, pp. 133-1140, 1997.

[68] M. Yu. Khlopov and A. S. Sakharov, "Cosmoparticle physics: as a probe for the theory of physical space-time," Gravitation and Cosmology, vol. 1, pp. 164-176, 1995.

[69] I. L. Buchbinder, S. D. Odintsov, and I. L. Shapiro, Effective Action in Quantum Gravity, IOP, Bristol, UK, 1992.

[70] I. L. Buchbinder, S. D. Odintsov, and I. L. Shapiro, "Nonsingular cosmological model with torsion induced by vacuum quantum effects," Physics Letters B, vol. 162, no. 1-3, pp. 92-96, 1985.

[71] I. L. Buchbinder and S. D. Odintsov, "Effective action in the de Sitter space with torsion," Acta Physica Polonica B, vol. 18, pp. 237-242, 1987.

[72] K. Bamba, S. Capozziello, S. Nojiri, and S. D. Odintsov, "Dark energy cosmology: the equivalent description via different theoretical models and cosmography tests," Astrophysics and Space Science, vol. 342, no. 1, pp. 155-228, 2012. 

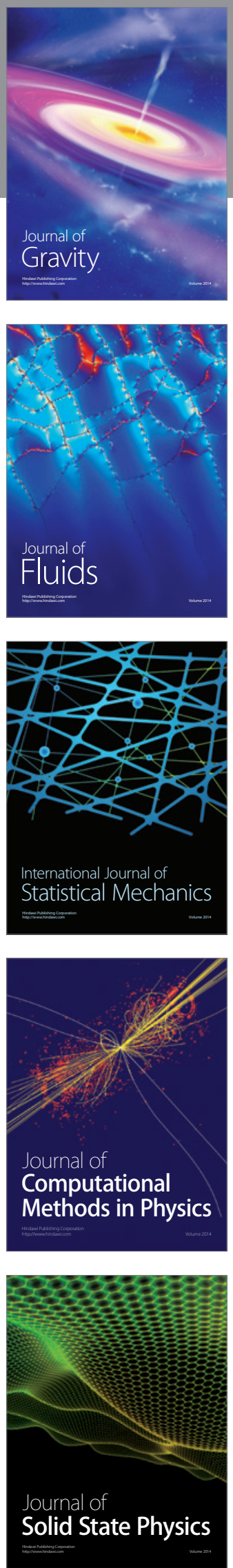

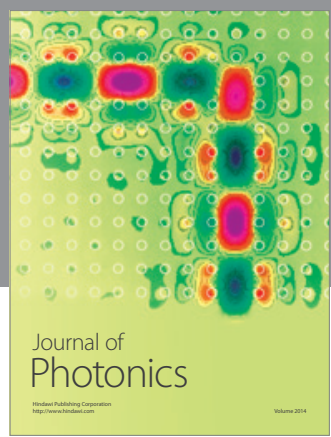

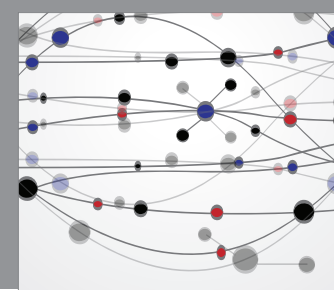

The Scientific World Journal

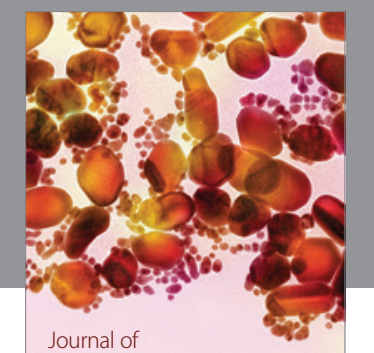

Soft Matter
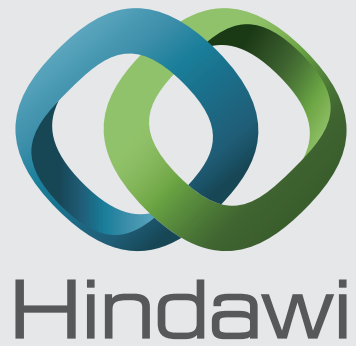

Submit your manuscripts at

http://www.hindawi.com
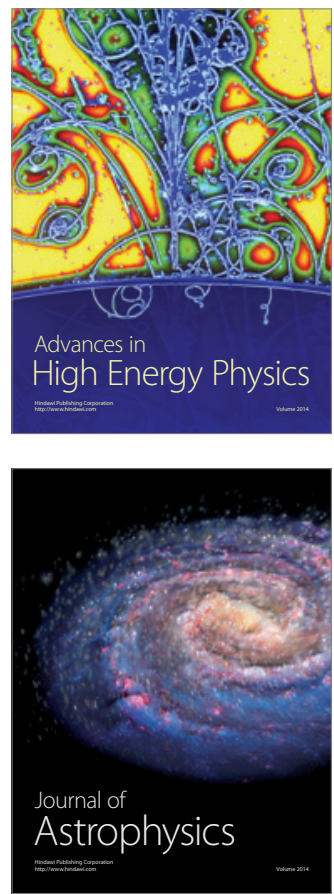
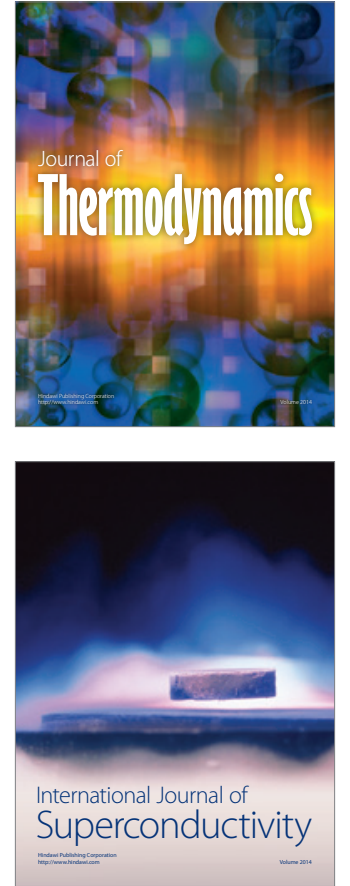
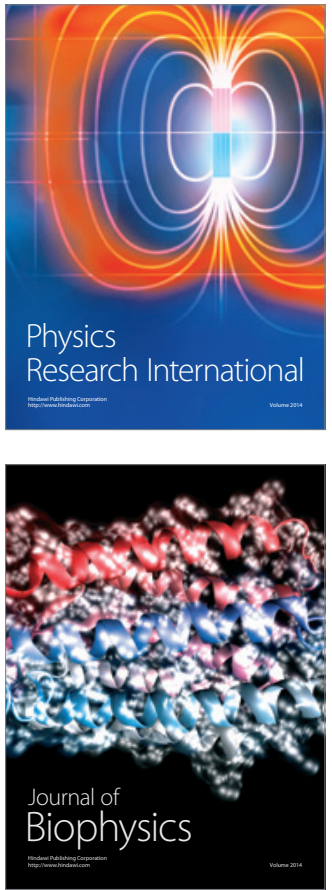
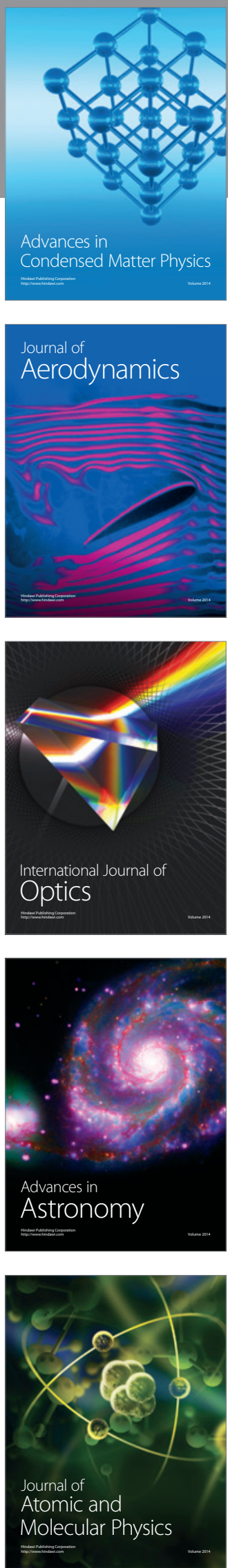